\title{
EXPERIENCE RATING WHEN THE COMPANY \\ AIMS TO INCREASE \\ THE VOLUME OF ITS BUSINESS
}

\section{HARALD BoHMAN \\ Stockholm}

An insurance company aims to increase the volume of its business. The volume is measured by the premium income during one year.

The company makes use of $N$ tariff groups.

$p_{n}=$ premium charged by the company per unit of insurance in tariff group $n$.

$q_{n}=$ premium per unit of insurance in tariff group $n$ corresponding to the statistical experience in the said group.

$L_{n} \quad=$ The size of the market in tariff group $n$. If all insurances in the group pay the same premium and if the company is able to cover the whole of its potential market then $L_{n}$ is equal to the number of policies. If the premium per policy is equal to $p_{n}$ times the sum insured and if the company is able to cover the whole of its potential market then $L_{n}$ is equal to the total sum insured.

$F_{n}(p)=$ the proportion of $L_{n}$ that actually buys insurance in tariff group $n$ if the company charges the premium $p$ per unit of insurance.

The total premium $P$ paid to the company for the portfolio will be equal to

$$
P=\sum_{1}^{N} L_{n} \cdot F_{n}\left(p_{n}\right) \cdot p_{n}
$$

Administration costs and claims paid by the company for the portfolio will be equal to

$$
A=\sum_{1}^{N} L_{n} \cdot F_{n}\left(p_{n}\right) \cdot q_{n}
$$

The aim of the company is to make $P$ as large as possible while $P$ is kept larger than $A$, or

$$
P=k \cdot A \quad \text { with } k \geqslant \mathrm{I}
$$


If we put $d P=$ o we get

$$
p_{n}=-\frac{F_{n}\left(p_{n}\right)}{F_{n}^{\prime}\left(p_{n}\right)} \text { for } n=\mathrm{I}, 2, \ldots, N,
$$

choosing the root of the equation which makes $p_{n} . F_{n}\left(p_{n}\right)$ as large as possible and if these values inserted in

$$
k=\frac{P}{A}
$$

give a $k$-value larger than I then $p_{1}, p_{2}, \ldots, p_{N}$ are a solution to the problem. Otherwise we procede as follows. Putting $p_{n}=q_{n}$ for $n=\mathrm{I}, 2, \ldots, N$ we get

$$
P=\Sigma L_{n} \cdot F_{n}\left(q_{n}\right) \cdot q_{n}=P_{0}
$$

which means that $P=A$. It is then possible to find a solution $p_{1}, p_{2}, \ldots, p_{N}$ which makes $P$ as large as possible while $k \geqslant \mathrm{I}$ and this $P$ is a fortiori larger than $P_{0}$. This solution may be found by some suitable numerical method.

We will not follow this mathematical model into more detail and draw only the following general conclusions from the preceding discussion. An insurance company which wants to fix its premium rates in such a way that the total premium income is both sufficient and as large as possible

shall probably not put $p_{n}=q_{n}$ for $n=\mathrm{I}, 2, \ldots, N$.

Making the premium income as large as possible means here of course what is achievable by a mere change of the premium rates. Nothing is said of other actions which the company may take with the same intentions or actions taken by competing companies. Such effects are not studied with the aid of the mathematical model described here. 